\title{
ORIGINAL
}

\section{Successful localization of ectopic ACTH-secreting bronchial carcinoid by selective pulmonary arterial sampling}

\author{
Miho Sugiyama $^{1) *}$, Toru Sugiyama1)*, Mina Yamaguchi' ${ }^{1)}$, Hajime Izumiyama ${ }^{1)}$, Takanobu Yoshimoto ${ }^{1)}$, \\ Mitsuhiro Kishino ${ }^{2)}$, Takumi Akashi ${ }^{3)}$ and Yukio Hirata ${ }^{1)}$ \\ ${ }^{1)}$ Department of Clinical and Molecular Endocrinology, Tokyo Medical and Dental University Graduate School, Tokyo 113-8519, Japan \\ 2) Department of Radiology, Tokyo Medical and Dental University Graduate School, Tokyo 113-8519, Japan \\ ${ }^{3)}$ Department of Pathology, Tokyo Medical and Dental University Graduate School, Tokyo 113-8519, Japan
}

\begin{abstract}
Among patient with ACTH-dependent Cushing's syndrome, about 10-20\% of those with ectopic ACTH syndromes (EAS) have occult or unknown tumors. Despite the intensive search for the culprit tumors by dynamic endocrine tests and imaging tests, it is often difficult to localize and confirm the source of occult ectopic ACTH secretion. We report a patient with EAS caused by a small bronchial carcinoid tumor, which was successfully localized by a selective pulmonary arterial sampling for the first time. A 69-year-old woman presented with typical Cushingoid features and elevated plasma ACTH and cortisol levels, which showed lack of circadian rhythm, no suppression by high-dose dexamethasone, and no response to CRH stimulation. No mass lesion was detected by pituitary MRI, and inferior petrosal sinus sampling showed no central to peripheral ACTH gradient. Although CT scan of the chest revealed a very small nodule in the right lung, it could not be confirmed by either somatostatin receptor scintigraphy or fluorodeoxyglucose positron emission tomography. Selective pulmonary arterial sampling of the wedged blood from a pulmonary artery branch affecting the nodule showed a marked ACTH gradient, and the lobectomy of the right middle lung resulted in dramatic decreases in plasma ACTH and cortisol levels. The resected tumor was diagnosed as a bronchial carcinoid tumor with positive immunostaining for ACTH. Thus, selective pulmonary arterial sampling, because of its more site-selective measurement of hormonal secretion, could be one of the useful tools to localize and confirm the ectopic ACTH production by a small pulmonary tumor.
\end{abstract}

Key words: Ectopic ACTH syndrome, Bronchial carcinoid, Selective pulmonary arterial sampling

CUSHING'S SYNDROME (CS) results from prolonged exposure to inappropriately excessive concentrations of circulating free glucocorticoids. The annual incidence of CS has been estimated at 0.7-2.4 per million population [1]. Among adrenocorticotropin (ACTH)-dependent CS, the majority (80-90\%) is due to pituitary adenomas (Cushing's disease) [1], whilst $10-20 \%$ is nonpituitary tumors, termed ectopic ACTH syndrome (EAS) [2, 3], whose source of ACTH is unidentified in about $10-20 \%$ of cases by any diagnostic means. About $50-80 \%$ of EAS have an ectopic source of ACTH within the chest, which comprise bronchi-

Received Jun. 23, 2010; Accepted Aug. 19, 2010 as K10E-192

Released online in J-STAGE as advance publication Sep. 28, 2010

Correspondence to: Toru Sugiyama, M.D., Ph.D., Department of Clinical and Molecular Endocrinology, Tokyo Medical and Dental University Graduate School, 1-5-45 Yushima, Bunkyo-ku, Tokyo 113-8519, Japan. E-mail: tsugiyama.cme@tmd.ac.jp

*These authors equally contributed to this report. al carcinoid tumor (> 25\%), small cell lung cancer ( 20\%), and adenocarcinoma ( 20\%) [4].

Since the treatment of choice for EAS is complete resection of the tumors, the correct diagnosis of localization and confirmation of the ectopic ACTH source is crucial. Despite the recent advance of diagnostic imaging and endocrine tests to correctly localize occult ACTH sources, there is no single imaging procedure accurate enough to confirm the localization of small and occult tumors [2-5]. The initial sensitivity of computed tomography (CT) and magnetic resonance imaging (MRI) for detection of EAS is $53 \%$ and $37 \%$, respectively, while functional imaging tests, such as ${ }^{111}$ In-octreotide scintigraphy (Octreoscan ${ }^{\circledR}$ ) and fluorodeoxyglucose positron emission tomography (FDGPET), have lower sensitivities of $47 \%$ and $35 \%$, respectively [6]. Since a single positive imaging study may represent a false-positive result, at least more than one positive study is necessary for confirmation of the 
ectopic ACTH source [3]. Furthermore, it remains uncertain whether the culprit tumors detected by any imaging studies are bona fide ectopic ACTH sources.

Selective venous blood sampling, especially inferior petrosal sinus sampling (IPSS) and adrenal vein sampling, are currently recognized as gold-standard tests for the localization of pituitary ACTH-producing tumors and aldosterone-producing tumors, respectively [1]. However, preoperative venous sampling from the effluent veins of the culprit tumors has not been successful for localization of occult EAS in most cases thus far reported [7-9]. We herein describe for the first time a case of EAS caused by a small bronchial carcinoid tumor, which could not be confirmed by any functional imaging tests, but was successfully localized by a selective pulmonary arterial sampling by demonstration of a marked ACTH gradient in the "wedged" blood in the branch of a pulmonary artery affecting the culprit lesion.

\section{Case report}

A 69-year-old woman with a two-month history of general fatigue was admitted to a hospital because of diabetes mellitus (HbA1c 10.3\%). She presented with moon face, acne and diffuse skin pigmentation, and was found to have hypertension $(144 / 86 \mathrm{mmHg}$ ) and hypokalemia (2.2 $\mathrm{mEq} / \mathrm{L})$. Initial endocrine examinations revealed elevated plasma ACTH $(205 \mathrm{pg} / \mathrm{mL})$ and serum cortisol (47.0 $\mu \mathrm{g} / \mathrm{dL})$ levels, and markedly increased urinary free cortisol excretion (4300 $\mu \mathrm{g}$ /day). Her serum cortisol level was not suppressed by overnight low-dose (1 mg) dexamethasone suppression test (DST). Because of her severe manic-depressive symptoms, she started to undergo treatment with metyrapone. She was referred to our hospital for the diagnostic workup for ACTH-dependent Cushing's syndrome.

Under treatment with metyrapone (1 g), her elevated levels of plasma ACTH (106 pg/mL) and cortisol $(21.6 \mu \mathrm{g} / \mathrm{dL})$ lacked circadian rhythm, and responded to neither overnight high-dose (8 mg) DST nor CRH stimulation. Her tumor markers, such as carcinoembryonic antigen, neuron-specific enolase and pro-gastrin-releasing peptide, were all within normal range.

Any mass lesion of the pituitary was not detected by pituitary MRI. Abdominal CT scan revealed no abnormal mass lesions except for bilateral adrenal enlargement. CT scan of the chest revealed a very small nodule $(7 \times 4 \mathrm{~mm})$ in the right middle lobe (segment 5 )

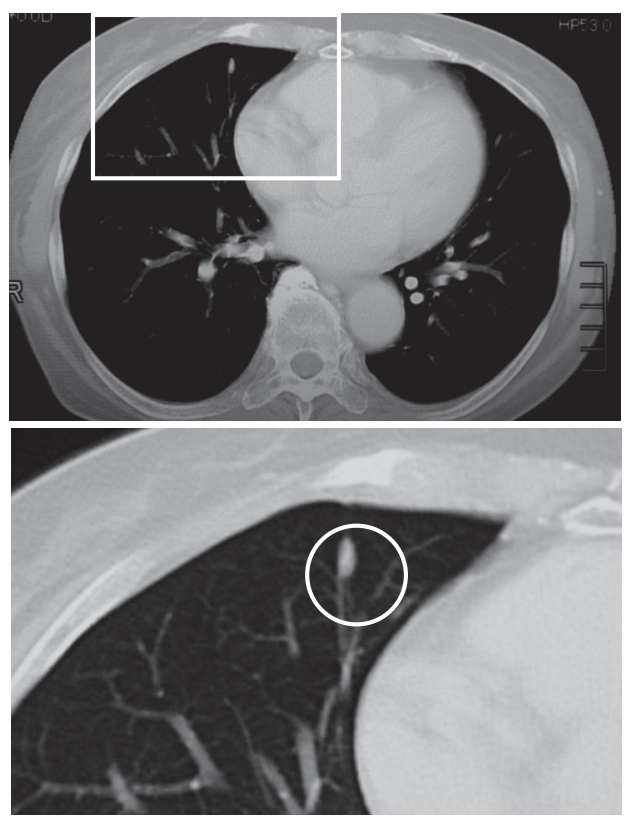

Fig. 1 Computed tomography (CT) scan of the chest. (Upper panel) Right middle lobe of the lung as indicated by the box. (Lower panel) A close-up view showing a nodule (7 $\times 4 \mathrm{~mm})$ in $\mathrm{rS}_{5}$ as indicated by a circle.

of the lung (Fig. 1), neither octreoscan nor FDG-PET, however, revealed any abnormal uptake in the lung. Bilateral IPSS showed no central to peripheral ACTH gradient before and after CRH stimulation; ACTH levels $(\mathrm{pg} / \mathrm{mL})$ in the right IPS, left IPS, and femoral vein were 217, 260, and 243 before CRH, and 247, 271, and 186 after $\mathrm{CRH}$, respectively. These endocrine and imaging data were consistent with the diagnosis of EAS, whose ectopic source of ACTH secretion remained unknown.

The patient's hypercortisolemia was well controlled with metyrapone (1 g) and replacement with dexamethasone $(0.5 \mathrm{mg})$ for three years, but she relapsed with marked elevations of plasma ACTH $(618 \mathrm{pg} / \mathrm{mL})$ and serum cortisol $(64.3 \mu \mathrm{g} / \mathrm{dL})$. Considering the small oval nodule in the right lung which did not change in its size and shape by repeated chest CT scanning during three years follow-up period and no evidence of elevated tumor markers related to other lung cancers, we reasoned that she is most likely to have an indolent bronchial carcinoid tumor because it is the most common and elusive ectopic ACTH source [5, 7]. After obtaining detailed informed consent from the patient, a selective pulmonary arterial sampling was performed to determine whether the small nodule in the right lung is responsible for the ectopic ACTH source. 


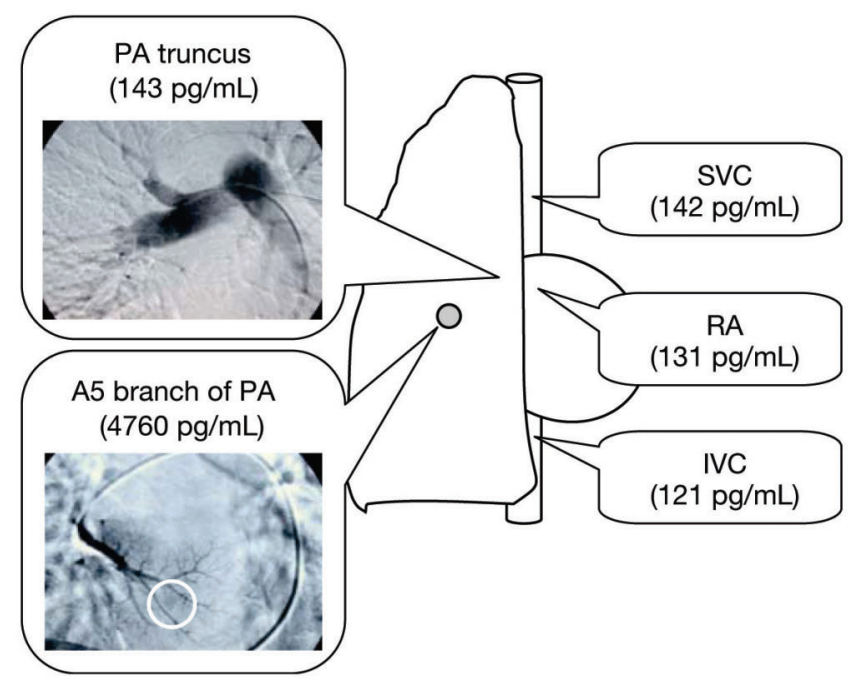

Fig. 2 Selective pulmonary artery samplings. Plasma ACTH levels in blood samples obtained from various sites are shown. Angiograms of the right pulmonary truncus (upper panel) and medial branch (A5) of the right pulmonary artery (lower panel) are shown with tumor stain in the right middle lobe $\left(\mathrm{rS}_{5}\right)$ as circled. Abbreviations used: SVC: superior vena cava, RA: right atrium of heart, IVC: inferior vena cava, PA: pulmonary artery.
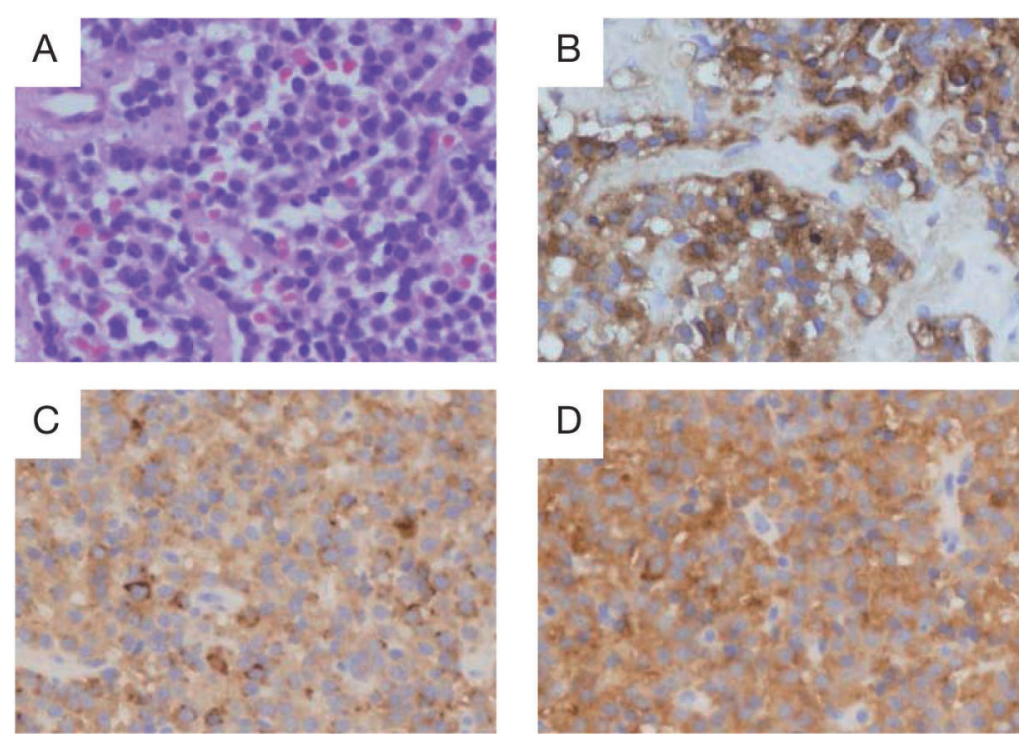

Fig. 3 Histopathological and immunohistochemical study of the resected pulmonary tumor. (A) Bronchial carcinoid tumor on microscopic examination (H\&E), and positive immunostaining for (B) ACTH, (C) chromogranin A, and (D) synaptophysin. Magnification (× 300).

After a wedge pressure balloon catheter (6 Fr., CI-216, Harmac Medical Products, Inc., NY, USA) was introduced via the right femoral vein, the tip of the catheter was inserted into the medial branch of right pulmonary artery (A5) closest to the tumor site (S5), and "wedged"; a blood sample was selectively withdrawn from the "wedged" branch vessel affecting the tumor. A marked gradient of plasma ACTH levels in A5
(4760 pg/mL) to the pulmonary truncus (143 pg/mL) was observed (Fig. 2), definitely consistent with the ectopic ACTH production from the tumor. She underwent medial lobectomy of the right lung; the resected tumor was pathologically consistent with the diagnosis of bronchial carcinoid tumor; immunohistochemical study showed positive staining for ACTH, chromogranin A, and synaptophysin (Fig. 3). Postoperative course 
was uneventful. Just after the operation, her plasma ACTH $(8 \mathrm{pg} / \mathrm{mL})$ and serum cortisol $(3.4 \mu \mathrm{g} / \mathrm{dL})$ levels fell dramatically, which necessitated her for glucocorticoid replacement (Fig. 4). A half-year later, her plasma ACTH $(<5 \mathrm{pg} / \mathrm{mL})$ and serum cortisol $(0.2 \mu \mathrm{g} /$ $\mathrm{dL}$ ) levels remained low with dexamethasone $(0.5 \mathrm{mg})$ replacement, but her Cushingoid appearance gradually disappeared.

\section{Discussion}

Ectopic ACTH-producing bronchial carcinoid, although accounting for only $1 \%$ of CS, is the most common cause of the occult EAS (37-57\%) [1, 2, 4]. Hormonally active bronchial carcinoids causing overt CS are less than $10 \%[10,11]$, but they are usually too small and slow-growing to be detected by the diagnostic imaging tests, such as plain X ray, CT scan, and MRI [6, 12]. Identification and localization of the source of ectopic ACTH secretion from the tumor, therefore, is challenging for both endocrinologists and radiologists.

The present case with typical Cushingoid features had elevated plasma ACTH and serum cortisol levels with lack of their circadian rhythm, no suppression by high-dose DST, and no response to CRH stimulation, along with no evidence of pituitary tumor on brain MRI and negative IPSS. These endocrine and imaging data were all consistent with EAS. However, it was difficult to localize the ectopic ACTH source in our case, even if CT scan of the chest detected a very small nodule in the lung, because two functional imaging tests (octreoscan, FDG-PET) failed to detect any abnormal uptake in the lung.

Octreoscan has been used as a useful tool for localization of the ectopic ACTH source in patients with occult EAS in otherwise negative conventional imaging modalities. Neuroendocrine tumors, including bronchial carcinoids, with preferential expression of somatostatin receptor (SSTR) subtype 2 or 5 can be visualized by octreoscan [13]. However, about 20\% of bronchopulmonary carcinoid tumors do not express SSTR-2 and -5 [10], and the sensitivity of octreoscan for the detection of EAS (47\%) [6] limited its diagnostic utility.

Selective venous blood samplings have been widely recognized as the gold-standard tests for confirmation and localization of hormone-producing tumors, such as in Cushing's disease and primary aldoster-

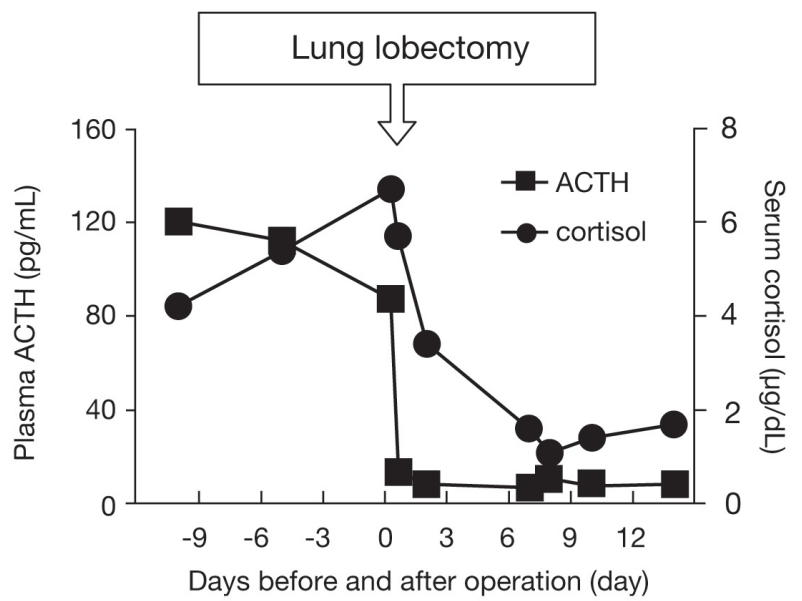

Fig. 4 Perioperative changes of plasma ACTH and serum cortisol levels. Plasma ACTH (ם) and serum cortisol (0) levels before and after the medial lobectomy of the right lung are shown. POD stands for postoperative day.

onism. In the cases of lung tumors, however, blood sampling from the pulmonary vein just downstream to the lesions, is apparently preferable, but not feasible, for the localization of the hormone secretion. In fact, pulmonary venous sampling and/or biopsy of the lung tumor have been attempted in certain EAS patients to confirm the ectopic ACTH source [2, 7-9, 14, 15]. However, selective blood sampling from the pulmonary vein via left-sided heart catheterization failed to localize the ectopic ACTH source in many EAS cases with bronchial carcinoids [7-9]. In addition, pulmonary venous sampling intraoperatively [16] or via thoracoscopic approach [17] is considered too invasive maneuvers to perform for the localization of the ectopic ACTH source in such patients with the suspected bronchial carcinoid tumors.

In the present case, the selective sampling of the "wedged" blood from a branch of the right pulmonary artery affecting the culprit pulmonary nodule in the right middle lobe of the lung has clearly revealed a distinct ACTH gradient. Aspiration of the "wedged" blood collected via backflow to the artery or via the capillary vessels around the tumor could accurately reflect the hormones ectopically secreted by the tumor. This procedure appears to be more site-selective for direct measurement of hormonal secretion, and less invasive via right-sided catheterization than pulmonary venous sampling via left-sided catheterization and/ or via intraoperative/thoracoscopic approach. Since wedging every segmental pulmonary artery (A1-A10) 
by a balloon catheter is theoretically and technically possible by the expertise radiologists, a selective pulmonary arterial sampling could be a useful and reliable tool to localize and confirm the ectopic ACTH source at least at the pulmonary segment level whenever CT scan or MRI detects a questionable small pulmonary tumor.

Surgical removal of a solitary tumor, such as bronchial carcinoid tumor, remains the only curative treatment of choice. The delay in localization of the source of EAS exposes the patients to unduly prolonged hypercortisolism, or requires bilateral adrenalectomy when medical therapy using steroidogenesis inhibitors has limited efficacy. Furthermore, various complications by hypercortisolism, including hypertension, diabetes, osteoporosis, and susceptibility to severe infection, increase mortality rate of EAS [1].

Therefore, the source of ectopic ACTH secretion should be accurately localized by using various imaging techniques. Anatomical imaging modalities, such as CT scan and MRI, although most commonly used to localize the tumors, are unable to distinguish ACTH-secreting tumors from nonsecretory ones. Moreover, many of the pulmonary tumors, especially carcinoid tumors, causing EAS are too small to be detected by conventional imaging tests $[6,12]$. Thus, the combination of anatomical and functional imaging tests is recommended for lesion confirmation [3]. Selective pulmonary arterial sampling should be taken into account as one of the diagnostic options to localize and confirm the ectopic ACTH source in such cases with a small, questionable pulmonary tumor with negative functional imaging tests as ours. However, a series of studies by this approach is needed in such suspected EAS patients.

\section{Acknowledgements}

This study was supported in part by the Grant-inaid from the Ministry of Education, Science, Sports and Culture, and the Ministry of Health, Welfare and Labor of Japan.

\section{References}

1. Newell-Price J, Bertagna X, Grossman AB, Nieman LK. (2006) Cushing's syndrome. Lancet 367: 16051617.

2. Isidori AM, Kaltsas GA, Pozza C, Frajese V, NewellPrice J, Reznek RH, Jenkins PJ, Monson JP, Grossman AB, Besser GM. (2006) The ectopic adrenocorticotropin syndrome: clinical features, diagnosis, management, and long-term follow-up. J Clin Endocrinol Metab 91: 371-377.

3. Zemskova MS, Gundabolu B, Sinaii N, Chen CC, Carrasquillo JA, Whatley M, Chowdhury I, Gharib AM, Nieman LK. (2010) Utility of various functional and anatomic imaging modalities for detection of ectopic adrenocorticotropin-secreting tumors. J Clin Endocrinol Metab 95: 1207-1219.

4. Isidori AM, Lenzi A. (2007) Ectopic ACTH syndrome. Arq Bras Endocrinol Metabol 51: 1217-1225.

5. Sookur PA, Sahdev A, Rockall AG, Isidori AM, Monson JP, Grossman AB, Reznek RH. (2009) Imaging in covert ectopic ACTH secretion: a CT pictorial review. Eur Radiol 19: 1069-1078.

6. Pacak K, Ilias I, Chen CC, Carrasquillo JA, Whatley M, Nieman LK. (2004) The role of $\left[{ }^{18} \mathrm{~F}\right]$ fluorodeoxyglucose positron emission tomography and $\left[{ }^{111} \mathrm{In}\right]$-diethylenetriaminepentaacetate-D-Phe-pentetreotide scintigraphy in the localization of ectopic adrenocorticotropin- secreting tumors causing Cushing's syndrome. J Clin Endocrinol Metab 89: 2214-2221.

7. Doppman JL, Nieman L, Miller DL, Pass HI, Chang R, Cutler GB Jr, Schaaf M, Chrousos GP, Norton JA, Ziessman HA, Oldfield EH, Loriaux DL. (1989) Ectopic adrenocorticotropic hormone syndrome: localization studies in 28 patients. Radiology 172: 115-124.

8. Drury PL, Ratter S, Tomlin S, Williams J, Dacie JE, Rees LH, Besser GM. (1982) Experience with selective venous sampling in diagnosis of ACTH-dependent Cushing's syndrome. Br Med J 284: 9-12.

9. Tremble JM, Buxton-Thomas M, Hopkins D, Kane P, Bailey D, Harris PE. (2000) Cushing's syndrome associated with a chemodectoma and a carcinoid tumour. Clin Endocrinol 52: 789-793.

10. de Matos LL, Trufelli DC, Neves-Pereira JC, Danel C, Riquet M. (2006) Cushing's syndrome secondary to bronchopulmonary carcinoid tumor: report of two cases and literature review. Lung Cancer 53: 381-386.

11. Gustafsson BI, Kidd M, Chan A, Malfertheiner MV, Modlin IM. (2008) Bronchopulmonary neuroendocrine tumors. Cancer 113: 5-21.

12. Loli P, Vignati F, Grossrubatscher E, Dalino P, Possa M, Zurleni F, Lomuscio G, Rossetti O, Ravini M, Vanzulli A, Bacchetta C, Galli C, Valente D. (2003) Management of occult adrenocorticotropin-secret- 
ing bronchial carcinoids: limits of endocrine testing and imaging techniques. J Clin Endocrinol Metab 88: 1029-1035.

13. Breeman WA, de Jong M, Kwekkeboom DJ, Valkema R, Bakker WH, Kooij PP, Visser TJ, Krenning EP. (2001) Somatostatin receptor-mediated imaging and therapy: basic science, current knowledge, limitations and future perspectives. Eur J Nucl Med 28: 14211429.

14. Rashid F, Riccio SA, Munk PL, Malfair D, Heran MK. (2009) Vertebroplasty for vertebral compression fractures secondary to Cushing's syndrome induced by an ACTH-producing bronchial carcinoid tumour. Singapore Med J 50: e147-150.
15. Ferguson ND, Poulin EC, Fornasier VL, Smyth HS, Ezzat S. (1998) A minimally invasive approach to the management of bronchial carcinoid tumors associated with ectopic Cushing's syndrome. Endocr Pathol 9: 249-253.

16. Raff H, Shaker JL, Seigert PE, Werner PH, Hazelrigg SR, Findling JW. (1995) Intraoperative measurement of adrenocorticotropin (ACTH) during removal of ACTH-secreting bronchial carcinoid tumors. J Clin Endocrinol Metab 80: 1036-1039.

17. Vu L, Theodore PR. (2005) Localization of a corticotropin-secreting tumor by thoracoscopic pulmonary venous sampling. N Engl J Med 353: 851-852. 